\title{
Shortest Path Queries in Rectilinear Worlds of Higher Dimension* (extended abstract)
}

\author{
Mark de Berg ${ }^{\dagger}$ \\ markdb@cs.ruu.nl
}

\author{
Marc van Kreveld ${ }^{\dagger}$ \\ marc@cs.ruu.nl
}

\author{
Bengt J. Nilsson ${ }^{\ddagger}$ \\ bengt@informatik.uni-freiburg.dbp.de
}

\begin{abstract}
In this paper, a data structure is given for higher dimensional shortest path queries. For a set of $n$ axisparallel boxes in $d$-space and a fixed target, it is possible with this structure to find a shortest rectilinear path from any point in $d$-space to this target, where the path does not cross any box. Alternatively, it is possible to find the length of the path. The metric considered is a generalization of the $L_{1}$-metric and the link metric, where the length of a path is its $L_{1}$-length plus some (fixed) constant times the number of tums on the path. The data structure uses $O\left((n \log n)^{d-1}\right)$ space to store, and a query takes $O\left(\log ^{d-1} n\right)$ time (plus the output size if the path must be reported). As a byproduct a solution to the single shot problem is obtained; the shortest path between two given points can be computed in time $O\left(n^{d} \log n\right)$.
\end{abstract}

\footnotetext{
${ }^{*}$ Work of the first author was supported by the Dutch Organization for Scientific Research (N.W.O.). Work of the first and second author was supported by the ESPRIT Basic Research Action No. 3075 (project ALCOM). Work of the third author was supported by the Deutsche Forschungs Gemeinschaft under Grant No. Ot 64/5-4.

${ }^{\dagger}$ Department of Computer Science, Utrecht University, P.O.Box $80.089,3508$ TB Utrecht, the Netherlands.

Institut für Informatik, Albert-Ludwigs-Universität, Rheinstr. 10-12, D-7800 Freiburg i. Br., Fed. Rep. of Germany.
}

Permission to copy without fee all or part of this material is granted provided that the copies are not made or distributed for direct commercial advantage, the ACM copyright notice and the title of the publication and its date appear, and notice is given that copying is by permission of the Association for Computing Machinery. To copy otherwise, or to republish, requires a fee and/or specific permission.

(3) 1991 ACM 0-89791-426-0/91/0006/0051 $\$ 1.50$

\section{Introduction}

The computation of shortest paths in some geometric space is a topic that has received considerable attention [1, 3], [6]-[9], [12]-[16], [18]-[25]. This is mainly due to the number of applications, such as in robotics, VLSI-design, and Geographical Information Systems (GIS). Shortest path problems have been studied in many different settings. In (almost) all settings, there is a finite set of obstacles, which have to be avoided by any legal path. Instances of shortest path problems include different restrictions on the obstacles, the use of different metrics, the dimension of the space, preprocessing version or single query, and more.

Most work has been done in planar settings, although recently there are some 3-dimensional results. We briefly review some of the more important results that relate to the work of this paper. (The time taken to report paths is omitted from the query time bounds; this is always linear in the number of segments of the path.)

Shamos[23] considers the problem of finding the shortest Euclidean path between two points inside a simple polygon with $n$ vertices. This problem is also studied by Chazelle[3] and by Lee and Preparata[15]. They solve it in $O(n \log n)$ time. With the linear time triangulation algorithm of Chazelle[4], this improves to $O(n)$ time. For $n$ parallel line segments, Lee and Preparata[15] obtain the same result. Lee[14] and Sharir and Schorr[24] consider an environment of arbitrary polygonal obstacles, and they present $O\left(n^{2} \log n\right)$ time solutions. For $k$ disjoint polygons with $n$ vertices in total, Reif and Storer[22] give an improved $O(n k+n \log n)$ time algorithm. De Rezende, Lee and $\mathrm{Wu}[10]$ start out with an environment of non- 
intersecting rectangles and a fixed target. They preprocess in $O(n \log n)$ time to a linear space data structure, such that rectilinear (i.e., axis-parallel) shortest paths can be retrieved in $O(\log n)$ time. Clarkson, Kapoor and Vaidya[7] study rectilinear paths among non-intersecting polygonal obstacles. Their result is improved by Mitchell[18], who gives an $O(n \log n)$ time algorithm. Furthermore, he presents a data structure of linear size, in which shortest path queries to a fixed target take $O(\log n)$ time.

In the link metric, the cost of a path is the number of turns the path makes, or alternatively, the number of segments on the path. The shortest link path is thus the path with the least number of turns. For this metric there are also a number of results. Lenhart et al.[16], Suri[25] and $\mathrm{Ke}[12]$ study several shortest link path problems inside simple polygons. Shortest link paths with respect to non-intersecting polygonal obstacles are considered by Mitchell, Rote and Wöginger[20]. They give an $O\left(n^{2} \alpha(n) \log ^{2} n\right)$ time algorithm. De Berg[8] studies rectilinear shortest link paths inside rectilinear polygons. He devises a data structure of size $O(n \log n)$, such that the rectilinear shortest link path between any two points can be found in $O(\log n)$ time.

In 3-dimensional space, shortest path problems are considerably harder. The general problem - shortest paths in the Euclidean metric among polyhedral obstacles - is known to be NP-hard[2]. The NPhardness result holds for any $L_{p}$-metric[1], where $p$ is a positive integer. Clarkson, Kapoor and Vaidya[7] give an algorithm for shortest rectilinear paths amidst non-intersecting rectilinear obstacles which runs in $O\left(n^{2} \log ^{3} n\right)$ time. The study of shortest paths on the surface of a polyhedron (convex or not) has received considerable attention[6, 19, 21, 24]. Best results sofar are of Chen and Han[6], who compute the shortest path between two points in $O\left(n^{2}\right)$ time.

In this paper we solve the following shortest path problem in arbitrary dimensional space. Given a set $S$ of $n$ possibly intersecting axis-parallel boxes (hyperrectangles) in $d$-space, a fixed target (point), and a nonnegative real $C$. Build a data structure such that for any point in $d$-space, the shortest path to the target can be found efficiently. The path may not intersect any of the boxes, and should be shortest in the combined metric. In this metric, the length of a path is the sum of its $L_{1}$-length, and $C$ times the number of turns in the path. Notice that if $C=0$, then the data structure answers shortest path queries in the $L_{1}$-metric, and if $C=\infty$, then the data structure answers shortest path queries in the link metric (i.e. paths with minimal number of links are found). See Figure 1 for examples of shortest rectilinear paths in the $L_{1}$-metric, the link metric, and the combined metric (for some value of $C$ ).

Let us motivate this metric from practical point of view. If we plan the motion of a mobile robot, we do not want it to make many turns, because making a turn involves slowing down, turning, and gaining speed again. Therefore the $L_{1}$-metric may be insufficient to plan shortest paths of robots. However, one also doesn't want the path with the least number of turns, because this path could make a large detour. A combination of the $L_{1}$-metric and link metric seems to be natural.

The problem we study here has been studied before in the plane in [9]. A data structure of size $O(n \log n)$ has been given that answers queries in $O(\log n)$ time. We generalize the approach used in that paper to higher dimensions, and use some of the same ideas. But in higher dimensions, a number of new problems appear that require new solutions. This leads us to an $O\left((n \log n)^{d-1}\right)$ space structure, in which queries take $O\left(\log ^{d-1} n\right)$ time (in $d$-space). Additionally, it takes $O(k)$ time to report a shortest path with $k$ segments. Preprocessing takes $O\left(n^{d} \log ^{d-2} n\right)$ time.

An immediate consequence of our work is a relatively simple algorithm for shortest rectilinear paths (in the combined metric) between two given points in a scene with $n$ boxes in $d$-space. It runs in $O\left(n^{d} \log n\right)$ time. This result is interesting in its own right. It implies that the shortest path problem in 'rectilinear worlds' of higher dimension is solvable in polynomial time, whereas the shortest path problem in 'Euclidean worlds' is NP-hard. As remarked before, this is also true for the $L_{1}$-metric. What saves us is having rectilinear obstacles and paths.

For several reasons, it is difficult to compare our work with previous work. First of all, the combined metric has not been used before (except in [9]). Secondly, we study the preprocessing version, whereas most au- 
thors study single shot shortest path problems (generally, the preprocessing versions are at least as difficult as the corresponding single shot problems). Thirdly, this paper gives the first results for shortest paths in dimensions greater than three (and on link distance in dimensions greater than two).

In Section 2, a number of definitions are given, and lemmas proved, upon which our work is based. A set of points, called induced points, is defined (as in Clarkson et al.[7]). They play a crucial role, as it is proved that any shortest path to the target will repeatedly visit induced points. Therefore, it is natural to split a shortest path query in two phases. In the first, it is computed to which induced point the shortest path from the query point should go first. The second phase consists of going from one induced point to the next, eventually reaching the target.

Section 3 studies the second phase; it solves the problem of finding the shortest path from any induced point to the target. The data structure obtained is basically a shortest path tree on the induced points, such that any node has an outgoing edge to that node that will lead to a shortest path to the target. (This approach is called 'continuous Dijkstra' in [19].) This will also lead to a solution to the single shot problem.

In Section 4, we consider how to find the induced point to which the query point should go first. This is needed for the query problem, contrary to the single shot problem. It results in a rather complicated recursive tree structure. The query proceeds by repeatedly searching in grids, ray shooting, and continuing in recursively defined structures. This leads to the main result of this paper.

The conclusions and directions for further research are given in Section 5.

In this extended abstract, the proofs of all lemmas are omitted. We refer to the full version for these proofs, the preprocessing of the slab tree, and some other details.

\section{Geometric preliminaries}

Let $S$ be a set of $n d$-boxes in $\mathbf{R}^{d}$, which are the obstacles of the problem. An $i$-face is defined to be an $i$-dimensional face of a $d$-box. Let $t$ be the target point, and let $C$ be a non-negative real which has as value the cost of making one turn.

Definition 1 The grid $G_{S}$ for a set $S$ of $d$-boxes is the arrangement of all axis-parallel hyperplanes that contain a facet of a $d$-box in $S$, together with the axisparallel hyperplanes that contain the target $t$. The lines of the grid $G_{S}$ are the closures of all 1-faces of $G_{S}$. The set $V_{S}$ of induced points of $S$ is that subset of the vertices of $G_{S}$ that lie on a $(d-2)$-face of some $d$-box in $S$ (including the target $t$ ).

The grid $G_{S}$ defines a subdivision of $\mathrm{P}^{d}$ into cells ( $d$ faces). Note that any cell either lies completely inside a $d$-box, or completely on the outside of it.

In 2-space, the set of induced points is the set of vertices of the rectangles, and in 3-space, all induced points lie on the edges of the boxes (at most $2 n+1$ induced points per edge). It is easily seen that the grid $G_{S}$ contains $O\left(n^{d}\right)$ vertices, whereas the set $V_{S}$ of induced points has size $O\left(n^{d-1}\right)$.

Definition $2 A$ directed polygonal chain $\overrightarrow{s_{1}} \overrightarrow{s_{2}} \ldots \overrightarrow{s_{m}}$ from a point $p$ to a point $q$ is called a rectilinear path from $p$ to $q$ if and only if each link $\vec{s}_{i}$ of the path is axis-parallel. A rectilinear path from $p$ to $q$ is called legal if and only if it does not intersect the interior of any $d$-box in $S$. A legal rectilinear path from $p$ to $q$ is called a simple step from $p$ to $q$ if and only if it consists of at most $d$ links, no two of which are parallel. $p$ is said to see $q$ if and only if there is a simple step from $p$ to $q$.

In the following we only consider legal rectilinear paths, so we omit the words 'legal' and 'rectilinear'.

Definition 3 A path $\Pi=\overrightarrow{s_{1}} \ldots \overrightarrow{s_{m}}$ from a point $p$ to $q$ has length $C \cdot(m-1)+\sum_{i=1}^{m}\left|\overrightarrow{s_{i}}\right|$, where $\left|\overrightarrow{s_{i}}\right|$ denotes the length of $\vec{s}_{i}$. The distance from $p$ to $q$ is the minimum of the lengths over all paths from $p$ to $q$.

Observe that the shortest path from one point to another is not necessarily unique. To see this, consider an obstacle-free environment in the plane. A shortest path is traced along either boundary segment pair of the rectangle defined by the two points. In fact, for 


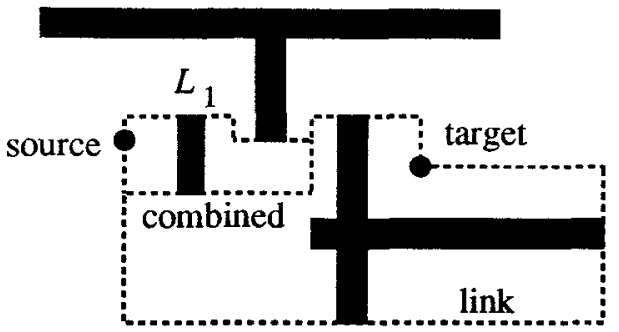

Figure 1: Shortest paths in the combined metric.

any value of $C$ and in any dimension $d \geq 2$, there can be an exponential number of 'different' shortest paths (exponential in $n$ ). Also notice that if $C=\infty$, then a shortest path in the combined metric will be a shortest path in the link metric, and if $C=0$, then it is the shortest path in the $L_{1}$ metric. See Figure 1 for an illustration of shortest paths in the combined metric.

It is possible to alter a path by a transformation called sliding. Let $\Pi=\overrightarrow{s_{1}} \ldots \overrightarrow{s_{m}}$ be a path, and let $\overrightarrow{s_{i}}$ and $\overrightarrow{s_{j}}$ $(i<j)$ be two parallel links of $\Pi$. Then, by changing the lengths of $\overrightarrow{s_{i}}$ and $\overrightarrow{s_{j}}$ with equal amounts, the subpath $\overrightarrow{s_{i+1}} \ldots \overrightarrow{s_{j-1}}$ can be moved into direction $+\overrightarrow{s_{i}}$ or $-\overrightarrow{s_{i}}$ while remaining axis-parallel. Notice that sliding the path into direction $-\vec{s}_{i}$ cannot make the path II longer.

With this transformation of a path, it immediatly follows that for any path $I I$ that has its endpoints on the grid $G_{S}$, there exists a new path between these endpoints which is no longer than $\Pi$, and only uses lines of the grid $G_{S}$. But we can prove something stronger, which justifies the use of induced points, and which is crucial for the solution and its efficiency.

Lemma 1 For any two induced points $p$ and $q$, there exists a shortest path $\Pi$ from $p$ to $q$, such that any $d$ consecutive links of $\Pi$ contain at least one induced point of $V_{S}$.

The above lemma will be used in the following way. For every induced point, we compute and store - as a part of the preprocessing - the next induced point on a shortest path to the target. This gives a tree rooted at $t$ on the $O\left(n^{d-1}\right)$ induced points, where every node (excluding the target) has one outgoing edge to its parent in the tree. Every path will (eventually) lead to the target. So from any induced point, the shortest path from the correspondin, node in the graph to the target. Furthermore, at every node we store the distance to the target.

It is natural to split a query in two parts. In the first, we compute the best induced point to which we can go with a simple step. (With 'best point' we mean the one that results in the shortest path.) The second part consists of following the path from the induced point that we just found, to the target (root) in the shortest path tree.

To decide upon which induced point to go to in the first simple step, it is comfortable to treat all possibilities of simple steps separately. The possibilities concern the directions of the links and their order. We make this more precise. A direction vector is a permutation of a non-empty subset of the base vectors of $\mathbf{R}^{d}$, which are prefixed by either + or - . Thus every simple step corresponds to a unique direction vector. So we can speak of a $\left(+x_{2},-x_{4},-x_{1}\right)$-simple step. When we only consider simple steps with one specific direction vector, we say that the direction vector is fixed. When we fix the direction vector, the term 'see' of course only refers to simple steps with the fixed direction vector. If the direction vector is fixed, then a simple step from one point to another is either unique or impossible.

Lemma 2 Let the direction vector be fixed, and let $p_{1}$ and $p_{2}$ be two induced points. If a point $q$ in $\mathrm{R}^{d}$ can take a simple step to both $p_{1}$ and $p_{2}$ (q sees both $p_{1}$ and $\left.p_{2}\right)$, and the path via $p_{1}$ has shorter total length, then for all points that can see both $p_{1}$ and $p_{2}$, the path via $p_{1}$ is shorter.

A point that can be seen, and which lies on a shortest path to the target (such as $p_{1}$ in the lemma), is called the best point (among some set of points that can be seen).

We conclude this section by discussing shortest paths and their length in the combined metric. A problem we have to face in the link metric and, hence, in the combined metric is that the distance function is not additive in the following sense. The distance from $p$ to $r$ via $q$ is not necessarily equal to the sum of the distances from $p$ to $q$ and from $q$ to $r$. The problem 

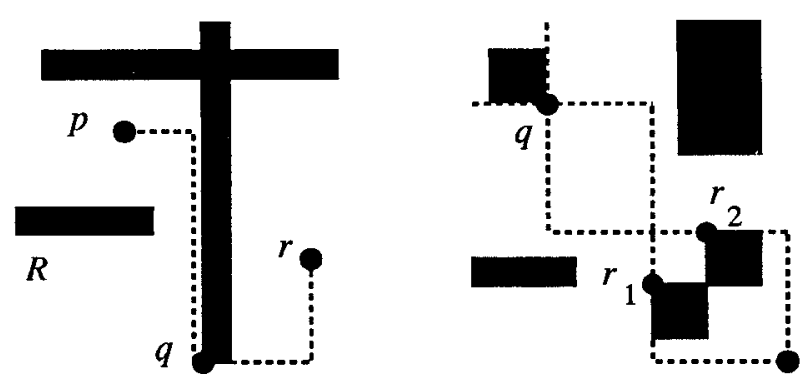

Figure 2: Difficulties in the combined metric.

is that in the latter case, the cost of a possible turn at point $q$ is not counted. This is shown in Figure 2. However, if the rectangle $R$ was not present, then the shortest path from $p$ to $r$ makes no tum at $q$. Even worse, it may be the case that a shortest path that arrives at some point $q$ horizontally, continues in a different way than a shortest path that arrives at the point $q$ vertically (see Figure 2), and the induced point to visit after $q$ is not unique.

We overcome these problems in the following way. We $d$-plicate every induced point $q$ in $V_{S}$, to get $d$ versions $q_{1}, \ldots, q_{d}$. Every one of the $d$ possible ways of arriving at (and leaving from) $q$ corresponds to one version. When a path enters $q$ with a link parallel to the $i^{\text {th }}$ coordinate axis, then it arrives at the version $q_{i}$. There are two ways to leave $q_{i}$. Either use a link parallel to the $i^{\text {th }}$ coordinate axis (simply continue in the same direction), or go to another version $q_{j}$ (which corresponds to making a turn at $q$ ). The distance from one version of $q$ to another version is defined to be $C$, the cost of making a turn. With this extension the distance function becomes additive. It is easy to see that the number of induced points remains $O\left(n^{d-1}\right)$, and that any shortest path visits at most two versions of one point.

\section{The shortest path graph}

In this section we concentrate on the structure that allows us to trace the shortest path to the target from any induced point. This structure is a single target shortest path graph, which we call sp-tree for short.
Let the set $S$ of $n d$-boxes be given, and also the target $t$ and the non-negative real $C$. The $d$-boxes and the target together define a grid $G_{S}$ with $O\left(n^{d}\right)$ vertices, and a set $V_{S}$ of $O\left(n^{d-1}\right)$ induced points. The sp-tree we aim to construct has one node for every induced point. With this node the distance to the target should be stored. Furthermore, a node corresponding to some point $p$ should have one outgoing edge to a node corresponding to a point $q$, where $q$ is the next induced point on a shortest path from $p$ to the target. There is a simple step from $p$ to $q$, and the links of this simple step should be stored explicitly with the edge in the graph. Recall that we actually use $d$ versions of each induced point, and there will be one node for each version in the sp-tree.

We do not want to compute the full simple step visibility graph on the induced points and then compute the sp-tree, since there can be $\Theta\left(n^{2 d-2}\right)$ edges in the full graph. Instead, we take the grid $G_{S}$, and show how to transform it into the sp-tree in $O\left(n^{d} \log n\right)$ time. Consider the grid $G_{S}$ to be a weighted graph on the $O\left(n^{d}\right)$ vertices of the grid in the following way. For every version of a vertex of the grid, there is a node in the graph (from now on, we will no longer make a distinction between a node in the graph and the version of the point it represents). Two nodes $p_{\imath}$ and $q_{j}$ in the graph are neighbours if and only if either $p=q$ and $i \neq j$, or $i=j, p$ and $q$ are neighbours in the grid $G_{S}$ and connected by an edge parallel to the $i^{\text {th }}$ coordinate axis. The graph is weighted in the usual way: every edge is weighted with the distance between the versions of the points. Notice that every node in the graph has degree at most $d+1$. The algorithm to change this graph into the sp-tree has three phases.

The first phase serves to delete all nodes that lie inside a $d$-box. Assume that $d \geq 3$. The grid $G_{S}$ is partitioned into roughly $n^{d-2}$ subgrids (slices) that contain roughly $n^{2}$ vertices each. Each slice is in fact a 2-dimensional object. Determine all relevant $d$ boxes (the ones that properly intersect the slice), and solve the 2-dimensional problem of finding all vertices that lie in the union of the at most $n$ relevant rectangles. This can easily be done by a plane sweep in $O\left(n^{2} \log n\right)$ time per slice. Deletion of one node from the graph can be done in constant time, because every node has constant degree. Hence, the first phase 
takes $O\left(n^{d}\right)$ time.

The second phase consists of the computation of the directed sp-tree on the (remaining) grid vertices. Note that the removal of the part of the grid lying inside $d$-boxes may have caused the graph to become disconnected. Trivially, we only have to consider the connected component that contains the target. Computing the directed sp-tree can be done with a priority breadth first search in $O\left(n^{d} \log n\right)$ time, where the priority is the distance to the target.

In the third phase all grid vertices that are not induced points, are removed. This is done in the following straightforward way. Take an induced point $p$, trace its shortest path until the next induced point $q$ is reached. Delete the outgoing edge of $p$, and insert an edge from $p$ to $q$. After doing this for every induced point, all grid vertices that are not induced points are removed from the graph. The resulting graph is the sp-tree on the induced points. The third phase also takes $O\left(n^{d}\right)$ time, because the path traced from any induced point to the next, contains at most a linear number of grid vertices.

We conclude with the main result of this section.

Theorem 1 Given a set $n$ of $d$-boxes, a target and a non-negative real $C$, the shortest path tree for the combined metric can be constructed in $O\left(n^{d} \log n\right)$ time and $O\left(n^{d}\right)$ space. The graph requires $O\left(n^{d-1}\right)$ space to store, and allows to retrieve for any induced point the distance to the target in constant time, and the path itself can be reported in $O(k)$ time, where $k$ is the number of links on the path. (For $d=2, O\left(n^{2}\right)$ time and space suffices to construct the tree, see [9].)

Corollary 1 Given a set of $n d$-boxes and two points, the shortest rectilinear path between them that does not cross any d-box can be computed in $O\left(n^{d} \log n\right)$ time in the $L_{1}$-metric, the link metric or the combined metric.

\section{Finding the first simple step}

In the previous section we solved the problem of finding the shortest path to the target from any induced point. However, a query point can be any point in $d$ space. In this section we consider how to find the first induced point on a shortest path to the target from any point $q$. It is sufficient to consider only grid vertices. With a solution to this, it takes a simple adaption to be able to handle any point in $d$-space. This is described at the end of this section. From any grid vertex, there is a shortest path to the target, such that it starts with a simple step to an induced point. (This follows from the proof of Lemma 1.)

We take the following approach. Fix the direction vector of the simple step and solve the problem using a recursive tree structure called $d$-dimensional slab tree. This is done for every direction vector, and a constant number of answers is found. An answer is the induced point that is the best if the path must start with a simple step with the specified direction vector. We also have the length of this path. Of all the paths (a constant number, namely one for each direction vector), the one resulting in a shortest path to the target (when the cost of the first simple step is added) is the actual shortest path.

Observe that if the locus approach is used (i.e., $d$-space is divided in regions, in which one induced point to go to with the first simple step is the best), every cell of the grid $G_{S}$ belongs completely to one region. Thus the subdivision has complexity $O\left(n^{d}\right)$. To facilitate the description, we assign to every induced point a unique colour (label). Then we wish to colour $d$ space with these $O\left(n^{d-1}\right)$ colours. The colour white is used as a special colour, indicating that no induced point can be reached at all (with this simple step). An explicit colouring has size $O\left(n^{d}\right)$ by the above argument, and below we show that an implicit colouring with a recursive data structure uses $O\left((n \log n)^{d-1}\right)$ space. This structure, called $d$-dimensional slab tree, is quite complicated. Therefore, we describe the 3dimensional version first, and then extend to higher dimensions. The 3-dimensional slab tree is shown schematically in Figure 3.

Assume without loss of generality that the direction vector is fixed to be $\left(-x_{3},-x_{2},-x_{1}\right)$. During our search in the slab tree we repeatedly have to answer questions of the form: how far can we go from a given point $q$ into a given axis-parallel direction without hitting an obstacle. For the moment, we assume 

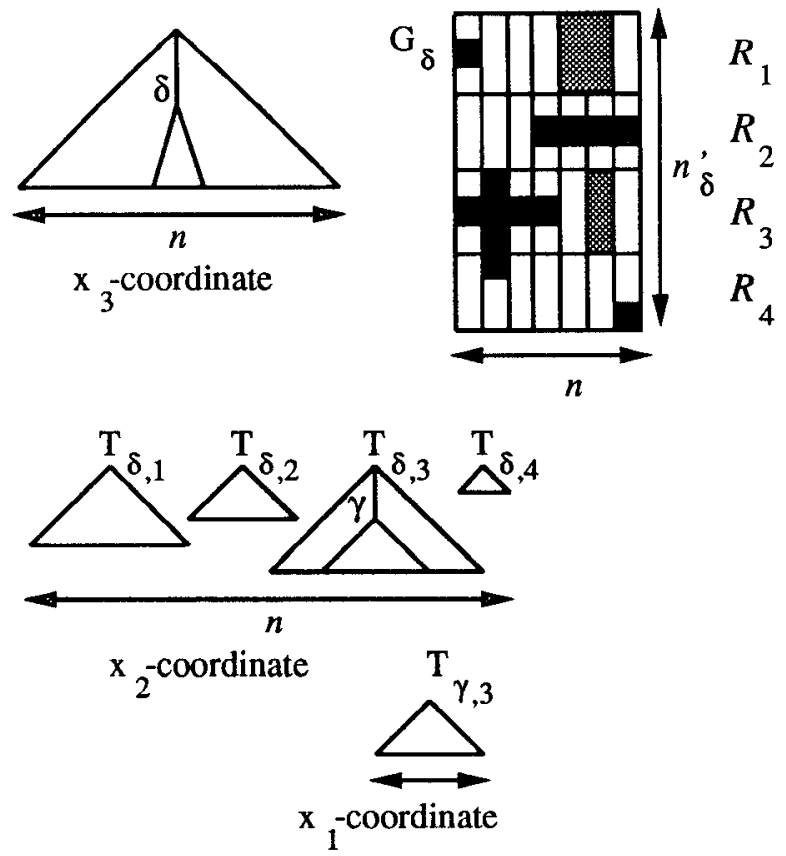

Figure 3: A 3-dimensional slab tree.

that we have a data structure for these axis-parallel ray shooting queries. Let the query point be $q$.

The 3-dimensional slab tree is a balanced binary tree with in the leaves the $O(n)$ different values of $x_{3^{-}}$ coordinates of the induced points. Every node $\delta$ corresponds to some $x_{3}$-interval (a 'slice' of 3-space). Let $n_{\delta}$ be the number of leaves in the subtree $T_{\delta}$ rooted at $\delta$. Any box $b$ either does not intersect the $x_{3}$-interval, or it has vertices in the $x_{3}$-interval (the so-called $x_{3^{-}}$short boxes at $\delta$ ), or it completely cuts through the slice (the so-called $x_{3}$-long boxes at $\delta$ ). Clearly, there are $O\left(n_{\delta}\right)$ boxes $x_{3}$-short at $\delta$. With $\delta$, we store a 2-dimensional grid $G_{\delta}$. In $G_{\delta}$, the $x_{3}$-coordinate is not used, and only a restricted set of $x_{2}$-coordinates, namely those that correspond to vertices of $x_{3}$-short boxes. This grid has size $n \times n_{\delta}^{\prime}$, where $n_{\delta}^{\prime} \leq 2 n_{\delta}$. In the projection of all $x_{3}$-short and $x_{3}$-long boxes onto the grid $G_{\delta}$, the first ones have all edges projected onto the set of edges of $G_{\delta}$, and the $x_{3}$-long boxes may intersect cells of $G_{\delta}$ with edges in $x_{1}$-direction. (In Figure 3, the $x_{3}$-short boxes are grey, and the $x_{3}$ long boxes are black.) $G_{\delta}$ can been seen as to consist of $n_{\delta}^{\prime}$ 1-dimensional subgrids of size $n$ called rows.
Every row $R$ is stored in two ways. First, we colour $R$ explicitly (i.e. we give each point of $R$ the colour of the best induced point), but only colours are used of induced points that lie in a different row $R^{\prime}$. (Note that we consider the $\left(-x_{3},-x_{2},-x_{1}\right)$ simple step, so $R^{\prime}$ must lie above $R$.) Secondly, we colour each row $R$ implicitly using a 2-dimensional slab tree, in which only the colours of induced points in the row $R$ itself are used.

Before we continue to describe the structure in more detail, we show how a query is done thusfar, to improve intuition for the structure. When querying, we shoot from the query point $q$ in $-x_{3}$-direction to see how far we can go with the first link. This gives an $x_{3}$-interval, with which we search in the 3-dimensional slab tree. The query interval selects $O(\log n)$ nodes that together span the $x_{3}$-interval (similar as for range trees). The query continues in the associated structure for any one of these nodes. Suppose $\delta$ is such a node. The query point $q$ projects somewhere on the grid $G_{\delta}$, and we know that it cannot be in the projection of an $x_{3}$-long or $x_{3}$-short box. (Otherwise, this node would not be selected by querying with the obstacle-free $x_{3}$ interval). With $q$, we query in both the grid, and in the 2-dimensional slab tree corresponding to the row in which $q$ lies. So we get two colours at $\delta$ corresponding to induced points which can both be reached. The best one is selected as answer to the query at $\delta$.

We continue to describe the grid $G_{\delta}$ more carefully. Let $m_{\delta}$ be the number of induced points in the subtree rooted at $\delta$. Clearly, $m_{\delta} \leq n \cdot n_{\delta}$. Let $p$ be any such induced point. Define for $p$ its histogram $H_{p}$ (or visibility region) to be that set of points in 3space that can see $p$ with respect to the simple step $\left(-x_{2},-x_{1}\right)$. Intuitively, consider the set of boxes and $p$ in 3-space, and shoot from $p$ in $+x_{1}$-direction. This gives an $x_{1}$-interval. With this interval, shoot in $+x_{2}$ direction, giving a histogram. It is easy to see that the query point $q$ can see $p$ if and only if the $x_{3}$-segment obtained from shooting with $q$ in $-x_{3}$-direction intersects with $H_{p}$. On the grid $G_{\delta}$, the histogram $H_{p}$ of $p$ is always obstructed by $x_{3}$-long boxes, and possibly by $x_{3}$-short boxes (depending upon whether the $x_{3}$ coordinate of $p$ lies in the $x_{3}$-interval of the $x_{3}$-short box). $H_{p}$ is split into two parts: $H_{p}^{1}$ is the part of $H_{p}$ that lies in the same row as $p$, and $H_{p}^{2}=H_{p}-H_{p}^{1}$. 
The part $H_{p}^{2}$ is used to colour the grid $G_{\delta}$ explicitly, and $H_{p}^{1}$ is used in the 2-dimensional slab tree corresponding to the row in which $p$ lies. The following lemma shows that the explicit colouring of $G_{\delta}$ using the parts $H_{p}^{2}$ is small of size.

Lemma 3 Any cell $c$ in $G_{\delta}$ has only one (real) colour (thus the colouring of $G_{\delta}$ has size $O\left(n \cdot n_{\delta}\right)$ ).

Next we describe more carefully what is done with the parts $H_{p}^{1}$ of histograms, which are restricted to the same row as the induced points $p$. Let $R_{i}$ be any one of the $n_{\delta}^{\prime}$ rows. Let $n_{\delta, i}$ be the number of different $x_{2}$-coordinates in $R_{i}$, and let $m_{\delta, i}$ be the number of induced points that project into $R_{i}$. Note that we have

$$
\sum_{i=1}^{n_{\delta}^{\prime}} n_{\delta, i}=n, \text { and } \sum_{i=1}^{n_{\delta}^{\prime}} m_{\delta, i}=m_{\delta} .
$$

For row $R_{i}$, a balanced binary tree $T_{\delta, i}$ is built on the $n_{\delta, i}$ different $x_{2}$-coordinates. For a node $\gamma$ in this tree, let $m_{\gamma}$ be the number of induced points in the subtree rooted at $\gamma$. Then $\gamma$ corresponds to some subgrid $G_{\gamma}$ of $G_{S}$ that lies inside $R_{i}$. This subgrid corresponds to the $x_{3}$-interval of $\delta$, the $x_{2}$-interval of $\gamma$ itself, and the full $x_{1}$-interval (the same as $G_{S}$, see Figure 4). The number of induced points in $G_{\gamma}$ is $m_{\gamma}$. The colouring of this grid is stored implicitly in a 1-dimensional tree on $x_{1}$-coordinate. In fact, we colour the line that bounds the grid $G_{\gamma}$ and has smallest $x_{2}$ - and $x_{3}$-coordinate (the fat line in Figure 4). One search in such a 1-dimensional tree suffices to find the best induced point of the subtree $T_{\gamma}$, or equivalently, of the subgrid $G_{\gamma}$.

\section{Lemma 4 The tree $T_{\gamma}$ has complexity $O\left(m_{\gamma}\right)$.}

We return to the query again. Let $q^{\prime}$ be the projection of $q$ in a row $R_{i}$. Then we shoot from $q^{\prime}$ in $-x_{2}$-direction, finding either an $x_{3}$-long box, or no obstruction at all in $R_{i}$. In both cases, an $x_{2}$-interval is found with which we search in $T_{\delta, i}$. Again, this results in a subset of $O(\log n)$ nodes, which form a partition of the $x_{2}$-interval. For each of these nodes, we search in the associated 1-dimensional tree.

Lemma 5 During a search with $q, O\left(\log ^{2} n\right)$ colours of induced points are found, and all these induced points are seen by $q$. The best point that can be seen from $q$ is among these.
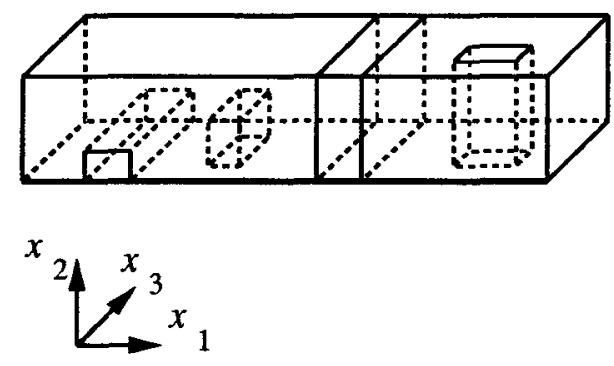

Figure 4: The subgrid $G_{\gamma}$ with intersecting boxes that are (from left to right) $x_{3}$-long, short, $x_{2}$ - and $x_{3}$-long, and $x_{2}$-long.

\section{Lemma 6 The}

3-dimensional slab tree uses $O\left((n \log n)^{2}\right)$ space to store.

This concludes the description of the 3-dimensional slab tree. The structure is extended to $d$ dimensions in the following way. A $d$-dimensional slab tree is a balanced binary tree on the different $x_{d}$-coordinates. Everey node $\delta$ has an associated structure, consisting of a $(d-1)$-dimensional grid $G_{\delta}$ of size $n \times \cdots \times$ $n \times n_{\delta}^{\prime}$, and for every one of the $n_{\delta}^{\prime}$ rows, there is a $(d-1)$-dimensional slab tree. The following results are extensions of the lemmas above.

Lemma 7 Any cell $c$ in $G_{\delta}$ has only one (real) colour (thus the colouring of $G_{\delta}$ has size $O\left(n^{d-2} \cdot n_{\delta}\right)$ ). A 1-dimensional tree $T_{\gamma}$, corresponding to a subgrid $G_{\gamma}$ with $m_{\gamma}$ induced points, has complexity $O\left(m_{\gamma}\right)$. During a search with $q, O\left(\log ^{d-1} n\right)$ colours of induced points are found, and all these induced points are seen by $q$, and the best induced point seen by $q$ is among these. The structure uses $O\left((n \log n)^{d-1}\right)$ space.

We can almost state our main theorem, but there are still two loose ends to take care of. First, there are the ray shooting structures that we need. Ray shooting in a fixed axis-parallel direction in a set of $n d$-boxes can easily be solved using $O\left(n \log ^{d-2} n\right)$ space and $O\left(\log ^{d-1} n\right)$ query time. For $d=2$, a simple planar point location structure suffices, and in higher dimensions, one segment tree reduces the dimension of the problem by one. This is well-known; see e.g. [17]. As we need to shoot $O\left(\log ^{i} n\right)$ times in $(d-i)$-space 
$(0 \leq i \leq d-2)$, this adds up to $O\left(\log ^{d-1} n\right)$ time for ray shooting. To get an efficient query time in the slab tree, fractional cascading[5] is applied to the 1-dimensional trees.

Lemma 8 A query in a d-dimensional slab tree with $a$ vertex of $G_{S}$ and for a fixed simple step can be answered in $O\left(\log ^{d-1} n\right)$ time.

A last loose end is that we only considered queries starting at a grid vertex, because for grid vertices we can prove that there is a simple step to an induced point. For an arbitrary point in $d$-space $(d \geq 3)$, however, it can be the case that it takes many links before the shortest path to the target joins an induced point. This problem can be overcome by doubling the grid, and adapting the path reporting algorithm. For details we refer to the full version.

The $d$-dimensional slab trees for each simple step, the ray shooting structures, and the shortest path graph together form the data structure that solves shortest path queries. In the full paper, we give an efficient algorithm for the preprocessing. The main result of this paper follows.

Theorem 2 Given a set of $n d$-boxes in $d$-space, $a$ target $t$ and a non-negative cost $C$, a data structure exists for shortest path queries to the target $t$ in the combined metric. The structure has size $O\left((n \log n)^{d-1}\right)$, and the distance from any point in $d$-space to the target can be found in $O\left(\log ^{d-1} n\right)$ time. The shortest path can be reported in additional $O(k)$ time, where $k$ is the number of links on the path. Preprocessing takes $O\left(n^{d} \log ^{d-2} n\right)$ time.

\section{Conclusions and directions for fur- ther research}

A shortest path problem has been studied, which brought two new aspects. First, a metric has been used that generalizes both the $L_{1}$ and link metric. The cost of a path was defined to be its $L_{1}$-length, plus some constant times the number of turns the path makes. The second important feature is that a shortest path problem is studied in arbitrary dimension. As far as we know, there are no other results on shortest paths in dimensions greater than three, and for link distance there are no results in dimensions greater than two.

This paper also shows that the general rectilinear shortest path problem can be solved in polynomial time in arbitrary dimension (in fact, in $O\left(n^{d} \log n\right)$ time), whereas the general Euclidean problem is already NP-hard in 3-dimensional space[2].

Many areas for future research lie open. First of all, it may be possible to improve our results. Futhermore, it is interesting to find other restricted cases of higher dimensional shortest path problems that are solvable in polynomial time. Finally, we believe that the generalization of our metric to non-rectilinear paths is worth studying.

\section{References}

[1] Canny, J.F., The Complexity of Robot Motion Planning. ACM Doctoral Dissertation Award, MIT Press, 1987.

[2] Canny, J., and J. Reif, New Lower Bound Techniques for Robot Motion Planning Problems. Proc. 29th FOCS (1987), pp. 49-60.

[3] Chazelle, B., A theorem on polygon cutting with applications. Proc. 23rd FOCS (1982), pp. 339-349.

[4] Chazelle, B., Triangulating a Simple Polygon in Linear Time. Proc. 31st FOCS (1990), pp. 220-230.

[5] Chazelle, B., and L.J. Guibas, Fractional Cascading: I. A Data Structuring Technique. Algorithmica 1 (1986), pp. 133-162.

[6] Chen, J., and Y. Han, Shortest Paths on a Polyhedron. Proc. 6th ACM Symp. on Comp. Geom. (1990), pp. 360-369.

[7] Clarkson, K., S. Kapoor, and P. Vaidya, Rectilinear Shortest Paths through Polygonal Obstacles in $O\left(n \log ^{2} n\right)$ Time. Proc. 3rd ACM Symp. on Comp. Geom. (1987), pp. 251-257.

[8] de Berg, M., On Rectilinear Link Distance. To appear in: Comp. Geom.: Theory and Applications.

[9] de Berg, M., M. van Kreveld, B. Nilsson, and M. Overmars, Finding Shortest Paths in the Presence of Orthogonal Obstacles using a Combined $L_{1}$ and Link Metric. SWAT 1990, Lect. Notes in Comp. Science 447 (1990), pp. 213-224. 
[10] de Rezende, P.J., D.T. Lee, and Y.F. Wu, Rectilinear Shortest Paths with Rectangular Barriers. Discr. \& Comp. Geom. 4 (1989), pp. 41-53.

[11] Dijkstra, E.W., A Note on Two Problems in Connection with Graphs. Numer. Math. 1 (1959), pp. 269271.

[12] Ke, Y., An Efficient Algorithm for Link Distance Problems. Proc. 5th ACM Symp. on Comp. Geom. (1989), pp. 69-78.

[13] Larson, R.C., and V.O. Li, Finding Minimum Rectilinear Distance Paths in the Presence of Barriers. Networks 11 (1981), pp. 285-304.

[14] Lee, D.T., Proximity and Reachability in the Plane. Ph.D. Thesis, University of Illinois, 1978.

[15] Lee, D.T., and F.P. Preparata, Euclidean Shortest Paths in the Presence of Rectilinear Barriers. Networks 14 (1984), pp. 393-410.

[16] Lenhart, W., R. Pollack, J. Sack, R. Seidel, M. Sharir, S. Suri, G. Toussaint, S. Whitesides, and C. Yap, Computing the Link Center of a Simple Polygon. Proc. 3rd ACM Symp. on Comp. Geom. (1987), pp. $1-10$.

[17] Mehlhorn, K., Data Structures and Algorithms 3: multi-dimensional searching and computational geometry. Springer-Verlag, Berlin, 1984.

[18] Mitchell, J.S.B., An Optimal Algorithm for Shortest Rectilinear Paths Among Obstacles in the Plane. Abstracts of the 1st Canad. Conf. on Comp. Geom. (1989), p. 22.

[19] Mitchell, J.S.B., D.M. Mount, and C.H. Papadimitriou, The Discrete Geodesic Problem. SIAM J. Comput. 16 (1987), pp. 647-668.

[20] Mitchell, J.S.B., G. Rote and G. Wöginger, Computing the Minimum Link Path Among a Set of Obstacles in the Plane. Proc. 6th ACM Symp. on Comp. Geom. (1990), pp. 63-72.

[21] O'Rourke, J., and C. Schevon, Computing the Geodesic Diameter of a 3-Polytope. Proc. 5th ACM Symp. on Comp. Geom. (1989), pp. 370-379.

[22] Reif, J., and J.A. Storer, Shortest Paths in Euclidian Space with Polyhedral Obstacles. Techn. Rep. CS85-121, Comp. Science Dept., Brandeis University, Waltham, MA, 1985.

[23] Shamos, M.I., Computational Geometry. Ph.D. Thesis, Yale University, New Haven, CN, 1978.
[24] Sharir, M., and A. Schorr. On Shortest Paths in Polyhedral Spaces. SIAM J. Comput. 15 (1986), pp. 193215.

[25] Suri, S., Minimum Link Paths in Polygons and Related Problems. Ph.D. Thesis, Johns Hopkins University, 1986. 